\title{
Considering racial and ethnic preferences in communication and interactions among the patient, family member, and physician following diagnosis of localized prostate cancer: study of a US population
}

This article was published in the following Dove Press journal:

International Journal of General Medicine

16 June 2011

Number of times this article has been viewed

\begin{abstract}
Sun Hee Rim'
Ingrid J Hall'

Megan E Fairweather ${ }^{2}$

Catherine R Fedorenko²

Donatus U Ekwueme'

Judith Lee Smith'

lan MThompson ${ }^{3}$

Thomas E Keane ${ }^{4}$

David F Penson ${ }^{5}$

Carol M Moinpour ${ }^{2}$

Steven B Zeliadt ${ }^{2,6}$

Scott D Ramsey ${ }^{2}$

'Division of Cancer Prevention and Control, Centers for Disease

Control and Prevention, Atlanta,

GA, USA; ${ }^{2}$ Public Health Sciences

Division, Fred Hutchinson Cancer

Research Center, Seattle, WA, USA;

${ }^{3}$ Department of Urology, University of

Texas Health Science Center at San

Antonio, TX, USA; ${ }^{4}$ Urology Services,

Medical University of South Carolina,

Charleston, SC, USA; ${ }^{5}$ Vanderbilt

Institute for Medicine and Public

Health, Vanderbilt University Medical

Center, Nashville, TN, USA; ${ }^{6} \mathrm{Health}$

Services Research and Development

Center of Excellence, VA Puget Sound

Health Care System, Seattle, WA, USA
\end{abstract}

Correspondence: Sun Hee Rim

Division of Cancer Prevention

and Control, Centers for Disease Control

and Prevention, 4770 Buford Highway NE,

MS K-55, Atlanta, GA 3034I-3724, USA

Tel +I 7704883252

Fax +I 7704884639

Email srim@cdc.gov

\begin{abstract}
Prostate cancer is the most commonly diagnosed cancer among American men. The multiple treatment options for localized prostate cancer and potential side effects can complicate the decision-making process. We describe the level of engagement and communication among the patient, family member, and physician (the decision-making "triad") in the decision process prior to treatment. Using the Family and Cancer Therapy Selection (FACTS) study baseline survey data, we note racial/ethnic variations in communication among the triad. Sensitivity to and awareness of decision-making styles of both the patient and their family member (or caregiver) may enable clinicians to positively influence communication exchanges about important clinical decisions.
\end{abstract}

Keywords: decision-making, treatment-related decisions, ethnicity

\section{Introduction}

Each year, approximately 186000 men are diagnosed with prostate cancer ${ }^{1}$ making it the most prevalent cancer among men in the USA. Once diagnosed, men with prostate cancer face difficult choices about their care given the lack of clinical consensus about prostate cancer management and the uncertainty of benefits from each treatment option. ${ }^{2}$

Without definitive clinical guidelines and strong evidence to support one treatment over another, most professional organizations encourage a shared decision-making approach in which a patient and his health care provider interact to determine the best management for the patient. However, due to the many treatment options available and potential mild to severe side effects of each treatment modality, patients may often receive complex and sometimes conflicting information from several sources about treatment and quality of life posttreatment.

In addition to recommendations from physicians, patient decisions and preferences are also often influenced by family members and other caregivers. ${ }^{3}$ Cultural differences in communication style may play a role in whether, and to what extent, patients discuss treatment decisions with spouses/caregivers. ${ }^{4,5}$ Our aim is to describe racial/ethnic tendencies in the level of engagement and communication among the patient, family member, and physician (the decision-making "triad") prior to selecting a treatment. 


\section{Method}

We analyzed data from the Family and Cancer Therapy Selection (FACTS) study, a multisite study designed to prospectively examine the treatment decision-making process of patients newly diagnosed with localized prostate cancer, along with their family members and their physicians. Men with incident localized prostate cancer $(n=240)$ and their family members $(n=193)$ were recruited at diagnosis from urology practice sites in California, South Carolina, and Texas and invited to participate in separate take-home baseline surveys prior to treatment. Urologists also completed a brief survey after the treatment planning visit. More details of the survey and study design have been described previously. ${ }^{6}$ All study methods and materials were reviewed and approved by Institutional Review Boards at the Centers for Disease Control and Prevention (CDC), the Fred Hutchinson Cancer Research Center study coordinating center, and at each recruitment site.

\section{Analysis}

For this analysis, patients were not included if they did not have a corresponding family member. Therefore, all 193 family members were paired with patients. To cover all points of the decision-making triad (patient/provider/family member), patient-partner pairs were included in further analyses only if family members had contact with a provider, resulting in 161 response pairs for analysis.

Analyses were descriptive. We identified questions from patient and family member baseline surveys that illustrated the perceived role of the respondent and/or level of communication in the decision-making process among "triad" members, specifically between the patient and the family member, patient and the physician, and the family member and the physician. We describe differences in response by race and ethnicity using SAS version 9.2 (SAS Institute, Inc, Cary, NC).

\section{Results}

Due to the small number of African-American and Hispanic patients and family members participating in this survey (Table 1), we present only descriptive results. Overall, more men were white $(71 \%)$, were married or living with a partner $(84 \%)$, and were in a higher (over US\$75 000) income bracket (49\%). Sixty-five percent were aged 60 and older. Most (93\%) of the participating family members were spouses/partners. Most (95\%) men had some form of health insurance and about $50 \%$ had at least a college education (Table 1).
Table I Demographic characteristics of prostate cancer patients and their family members

\begin{tabular}{|c|c|c|c|c|}
\hline \multirow[t]{2}{*}{ Total } & \multicolumn{2}{|c|}{ Patients } & \multicolumn{2}{|c|}{$\begin{array}{l}\text { Family } \\
\text { members }\end{array}$} \\
\hline & $\mathbf{N}$ & $\%$ & $\mathbf{N}$ & $\%$ \\
\hline Total & 240 & & 193 & \\
\hline \multicolumn{5}{|l|}{ Age } \\
\hline$<60$ & 85 & $35 \%$ & 100 & $52 \%$ \\
\hline $60-64$ & 57 & $24 \%$ & 36 & $19 \%$ \\
\hline $65-69$ & 56 & $23 \%$ & 31 & $16 \%$ \\
\hline$\geq 70$ & 42 & $18 \%$ & 20 & $10 \%$ \\
\hline \multicolumn{5}{|l|}{ Sex } \\
\hline Female & & & 187 & $97 \%$ \\
\hline \multicolumn{5}{|l|}{ Relationship with patient } \\
\hline Wife/Partner & & & 179 & $93 \%$ \\
\hline Daughter/Son & & & 9 & $5 \%$ \\
\hline Other/Unknown & & & 5 & $3 \%$ \\
\hline \multicolumn{5}{|l|}{ Race } \\
\hline White & 170 & $71 \%$ & 134 & $69 \%$ \\
\hline Black & 38 & $16 \%$ & 24 & $12 \%$ \\
\hline Hispanic & 26 & $11 \%$ & 17 & $9 \%$ \\
\hline Other/Unknown ${ }^{\mathrm{a}}$ & 6 & $3 \%$ & 18 & $9 \%$ \\
\hline \multicolumn{5}{|l|}{ Insurance } \\
\hline Medicare & 93 & $39 \%$ & & \\
\hline Private (non-Medicare) & 96 & $40 \%$ & & \\
\hline VA/Military & 36 & $15 \%$ & & \\
\hline None/Unknown & 11 & $5 \%$ & & \\
\hline \multicolumn{5}{|l|}{ Employment } \\
\hline Full time & 104 & $43 \%$ & & \\
\hline Part-time/Self-employed & 45 & $19 \%$ & & \\
\hline Retired & 81 & $34 \%$ & & \\
\hline Unemployed/Unknown & 10 & $4 \%$ & & \\
\hline \multicolumn{5}{|l|}{ Income (USD) } \\
\hline$<\$ 40000$ & 68 & $28 \%$ & & \\
\hline$\$ 40000-\$ 74999$ & 48 & $20 \%$ & & \\
\hline$\geq \$ 75000$ & 117 & $49 \%$ & & \\
\hline \multicolumn{5}{|l|}{ Education } \\
\hline High school or less & 50 & $21 \%$ & 36 & $19 \%$ \\
\hline Some college & 65 & $27 \%$ & 64 & $33 \%$ \\
\hline College graduate & 59 & $25 \%$ & 62 & $32 \%$ \\
\hline Graduate degree & 61 & $25 \%$ & 30 & $16 \%$ \\
\hline \multicolumn{5}{|l|}{ Marital status } \\
\hline Married or living with a partner & 202 & $84 \%$ & 180 & $93 \%$ \\
\hline Other/Unknown & 38 & $16 \%$ & 13 & $7 \%$ \\
\hline
\end{tabular}

Notes: aPatients of "other/unknown" race were Asian $(n=6)$. Among family members of "other/unknown" race, twelve were Asian and six were of unknown race. Percentages may not all total $100 \%$ because of missing data.

Abbreviation: VA, Veteran Affairs.

Across all races, most ( $\geq 75 \%$ ) patients reported that the physician "definitely" involved them in the decisionmaking process, "definitely" discussed treatment options in an understandable way, or "definitely" encouraged questions about treatment options (Table 2). From the patient perspective, although some patients ( $58 \%$ across all races) found wife/partner preference for a particular treatment "very important," a greater proportion of African-American men in 
Table 2 Communication in decision-making process among the triad (patient, physician, family member) by race/ethnicity

\begin{tabular}{|c|c|c|c|}
\hline \multirow[t]{2}{*}{ Triad relationship } & \multicolumn{3}{|c|}{ Race/ethnicity } \\
\hline & $\begin{array}{l}\text { White } \\
(n=125)\end{array}$ & $\begin{array}{l}\text { African-American } \\
(n=19)\end{array}$ & $\begin{array}{l}\text { Hispanic } \\
(n=I 7)\end{array}$ \\
\hline \multicolumn{4}{|l|}{ Patient-physician $(n=173)$} \\
\hline Physicians "definitely" involved me in the decision process & $74 \%$ & $84 \%$ & $82 \%$ \\
\hline Physicians "definitely" discussed treatment options in an understandable way & $84 \%$ & $89 \%$ & $100 \%$ \\
\hline Physicians "definitely" encouraged me to ask questions about treatment options & $79 \%$ & $89 \%$ & $88 \%$ \\
\hline Physician recommendation "very important" & $86 \%$ & $89 \%$ & $94 \%$ \\
\hline \multicolumn{4}{|l|}{ FM-physician $(n=161)$} \\
\hline Physicians "definitely" discussed treatment options in an understandable way & $90 \%$ & $89 \%$ & $76 \%$ \\
\hline Physicians "definitely" encouraged me to ask questions about treatment options & $77 \%$ & $95 \%$ & $76 \%$ \\
\hline Communicated with physicians on my own & $12 \%$ & $21 \%$ & $0 \%$ \\
\hline FM "very comfortable" discussing cancer issues with physician & $85 \%$ & $79 \%$ & $82 \%$ \\
\hline \multicolumn{4}{|l|}{ Patient-FM $(n=16 \mathrm{I})$} \\
\hline Patient considers wife/partner preference for a particular treatment "very important" & $54 \%$ & $74 \%$ & $71 \%$ \\
\hline Patient considers close FM preference for a particular treatment "very important" & $21 \%$ & $47 \%$ & $24 \%$ \\
\hline FM discussed treatment options "very often" with patient & $51 \%$ & $26 \%$ & $41 \%$ \\
\hline FM attended physician visits "often" after diagnosis & $78 \%$ & $79 \%$ & $82 \%$ \\
\hline
\end{tabular}

Note: Hispanic denoted in addition to racial status.

Abbreviation: FM, family member.

particular (74\%) valued the importance of their wife/partner and a close family member's preference (47\%) (Table 2). Most ( $\geq 80 \%$ ) patients, regardless of race, considered recommendations from their doctor "very important."

Family members generally felt "very comfortable" discussing cancer issues with the physician and attended physician's visits "often" after patient diagnosis (Table 2). Much like the patients, a high proportion of family members also affirmatively reported that the physician "definitely" discussed treatment options in an understandable way and encouraged questions. However, African-American family members more frequently reported that physicians "definitely" encouraged them to ask questions about treatment options (African-Americans 95\%; whites 77\%; Hispanics 76\%). Family members reported discussing treatment options with patients "very often" among $26 \%$ of African-American, $41 \%$ of Hispanic, and $51 \%$ of white families. Although discussion occurred less often between the family member and physician alone (African-Americans $21 \%$; whites $12 \%$; Hispanics $0 \%$ ), a greater proportion of AfricanAmerican family members reported having independent conversations with the physician than whites.

Lastly, we assessed how family members viewed their role in treatment decision-making. Nearly all family members (97\%) "strongly agreed" their role was to listen and provide emotional support, and the majority of family members ( $82 \%$ ) "strongly agreed" their role was to help get information about cancer and treatment options (Figure 1). A greater proportion of Hispanic family members "strongly agreed" that their role was to arrange meetings with physicians and other health professionals (Hispanics 71\%, African-Americans 58\%, whites $52 \%$ ), as well as to help weigh pros and cons of each treatment option (Hispanics $88 \%$, whites $80 \%$, AfricanAmericans 74\%). Slightly more African-American (79\%) and Hispanic (71\%) than white (65\%) family members "strongly" felt their role was to help patients make a treatment decision. Our results were not statistically significant at $P<0.05$.

\section{Discussion}

Communication is a complex process, involving multilevel interactions, understanding of the content of discussion, and the relational aspects of cultures and behaviors, which are critical to the decision-making process and for ensuring comprehensive, quality cancer care and outcomes. ${ }^{7,8}$ Our data provide insight into the interaction and engagement of the patient, family member, and physician around treatment-related decisions for localized prostate cancer by race/ethnicity. Variations in communication and interaction styles among the triad of decision-makers can be important considerations for further investigations into providing culturally sensitive communication concerning prostate cancer treatment.

Results from our study show that, overall, most patients and family members felt that their physician "definitely" encouraged involvement in decision-making and that treatment options were discussed in an understandable way. Still, most patients highly regarded the opinions/recommendations of their physician, suggesting that the patient's treatment decision may ultimately have relied on the recommendation of their urologist. ${ }^{9,10}$ Although our data did not directly assess 


\section{Family member roles}

White $(\mathrm{N}=125) \quad$ African-American $(\mathrm{N}=19) \quad$ Hispanic $(\mathrm{N}=17)$

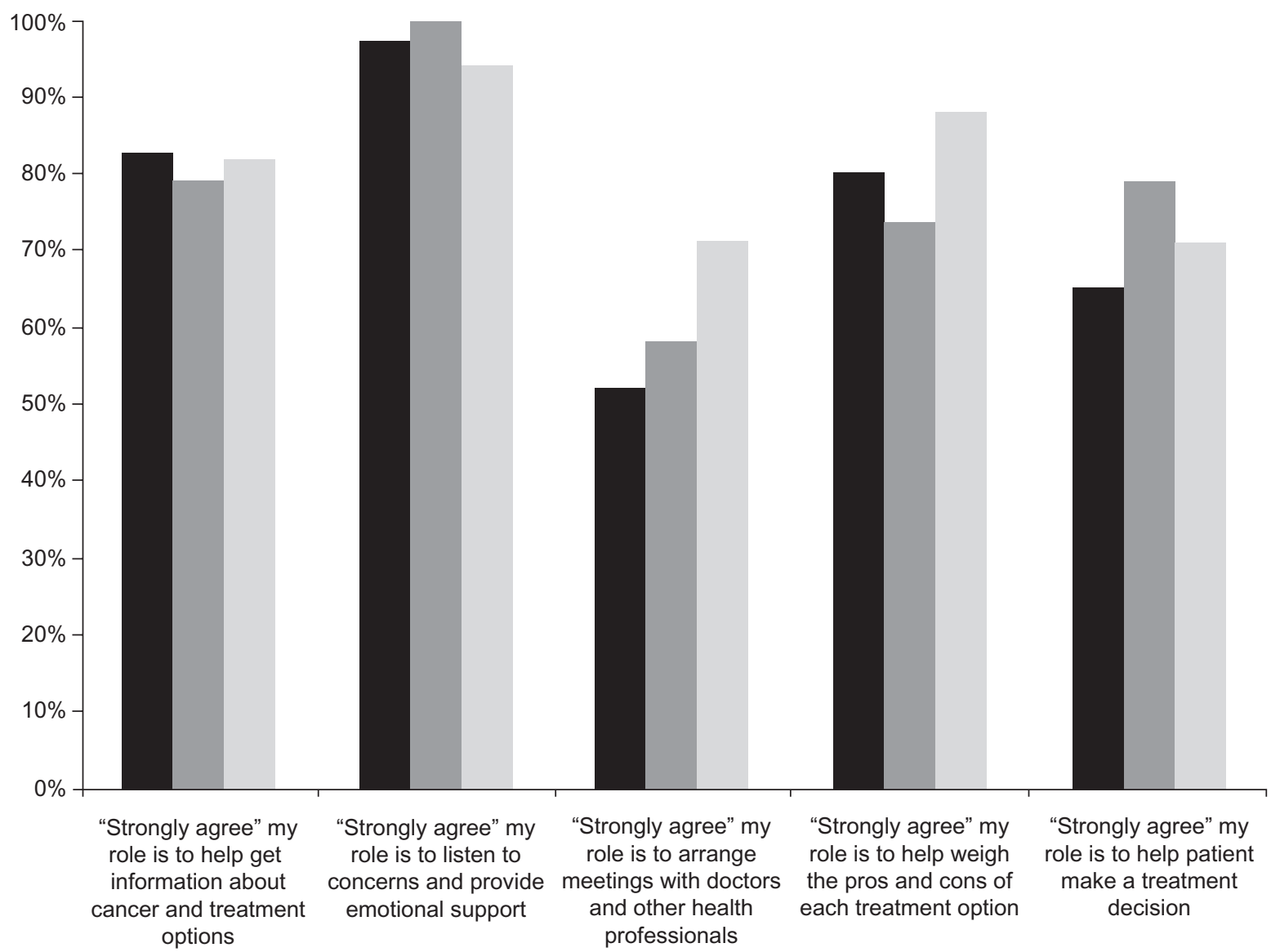

Figure I Perceived family member roles.

this dynamic, such behavior is not unusual given that physicians are often called upon for their expertise and experience and have historically been entrusted to make medical decisions in the best interest of the patient. ${ }^{11}$ While previously, men may have taken a more passive role in selecting their treatment ${ }^{12}$ following the guidance of their physician, more recent studies suggest that men prefer greater involvement/ collaboration in treatment decision-making for localized prostate cancer ${ }^{13-15}$ and tend to be more satisfied with their role in the decision-making given individualized information. ${ }^{16}$ Although men seem to prefer to be increasingly involved in their care, we observed that most men in our study continue to regard physician opinions highly. These interactions in the decision-making process are important to monitor as studies have shown the aggressiveness of the treatment (level of toxicity) selected for prostate cancer can often depend on the assertiveness of the managing physician and can differ from physician's predictions for patients' preferences. ${ }^{17,18}$
Additionally, treatment-specific decisions could over time increase the patient's decisional regret following prostate cancer treatment. ${ }^{19}$

Communicating and collaborating with family members (usually spouses) and other caregivers of patients with cancer also affect the cancer diagnosis and treatment experience. Lack of communication about prostate cancer between spouses has been shown to negatively impact patient-partner satisfaction and adjustment to treatment-related issues. ${ }^{20}$ Effective communication in the level of information provided and interpersonal behaviors between the physician-patient and family members, could improve satisfaction for both the provider and patient. ${ }^{21}$ Although it is debatable to what extent family members (spouse) influence a patient's final decision about treatment, a spouse's presence is important for the roles they play in obtaining information, providing emotional support, and aiding in decisions. Our data support that many family members strongly perceive responsibility 
in providing this type of caregiving assistance to the patient, in weighing the pros and cons of each treatment option, and in helping the patient make a treatment decision.

Race/ethnicity and cultural background have critical bearing on how cancer is discussed and processed individually and in partnership. ${ }^{4}$ Our findings showed there were racial/ ethnic variations in communication among the triad, although not statistically significant at $P=0.05$. African-American family members more frequently reported feeling encouraged to ask questions about treatment options and also tended to report having independent conversations with the physician compared with whites. Family members of African-American patients reported fewer discussions about treatment options with the patient and appeared to perceive a stronger role in decision-making than white family members. Comparatively, African-American patients also valued recommendations of their family member more than white patients. Although our data for this population are sparse, a similarly higher proportion of Hispanic family members compared with all other race/ethnicities "strongly agreed" their role was to support the patient by helping to arrange meetings with doctors and weigh the pros and cons of each treatment option.

Other studies that have characterized family involvement with prostate cancer treatment decisions among AfricanAmerican prostate cancer survivors have also shown that men particularly value their wives' opinions about the best treatment option and that family members generally play an important role in making decisions about the treatment the men receive.22 Our data seem to indicate similar findings. Furthermore, focus groups conducted by Williams et al found that African-American participants emphasized that effective communication involved "knowing" and "understanding" the patient and family individually so that communication could be tailored through this relationship. ${ }^{5}$ Also, African-American focus group participants (a mixture of caregivers and cancer survivors) preferred participating in the decision-making process as it equated to having a "sense of control." This cultural aspect may explain the higher level of participation we observed among African-American family members in engaging with the physician.

There are several limitations to this study. First, communication among the triad is a complex process and one that is difficult to comprehensively measure and accurately attribute. Our questions only serve as a proxy to the shared decisionmaking process and the content, depth, and level of discussion that should have appropriately taken place. Second, the sample size was rather small for African-American and Hispanic participants and did not allow for more detailed analyses of these subpopulations, and therefore the inferences that can be concluded are limited. As such, there is a possibility of selection bias, and these results should be replicated in a larger population-based study. Third, we were unable to compare the participants who responded to our survey with those who received it but chose not to complete the survey to assess the impact of nonresponse bias. Those who agreed to participate may be different from nonparticipants in their behaviors, attitudes, and interest in engaging in decision-making. Despite these limitations, our study is one of few studies that have characterized racial/ethnic differences in communication preferences from paired surveys. We believe that the results of this study provide helpful information about communication and interaction preferences among the triad of decision-makers.

Understanding the dynamics of partner communication and physician interaction is an important part of improving outcomes in clinical practice by aiding patients and their family members in making appropriate health- and treatmentrelated decisions. Considering the cultural, interpersonal, and decision-making styles of patients and their family members can help clinicians facilitate improved understanding of cancer management for their patients. Burkhalter and Bromberg previously outlined and advocated for further evaluation of cultural effects on patient-family member-physician communication. ${ }^{21}$ Our results suggest variation in prostate cancer treatment-related discussions can differ by race/ethnicity and may substantiate the need for further research on how cultural influences (including faith and beliefs) and communicative behavior affect discussions and decisions about cancer treatment and outcome.

\section{Acknowledgment}

This publication was supported by Cooperative Agreement Number 1-U48-DP-000050 from the Centers for Disease Control and Prevention, Prevention Research Centers Program, through the University of Washington Health Promotion Research Center.

The findings and conclusions in this report are those of the authors and do not necessarily represent the official position of the Centers for Disease Control and Prevention.

\section{Disclosure}

The authors report no conflicts of interest in this work.

\section{References}

1. US Cancer Statistics Working Group. United States Cancer Statistics: 1999-2007 Incidence and Mortality Web-based Report. Atlanta, GA: US Department of Health and Human Services, Centers for Disease Control and Prevention and National Cancer Institute; 2010. 
2. Wilt TJ, Shamliyan T, Taylor B, et al. Comparative Effectiveness of Therapies for Clinically Localized Prostate Cancer: Comparative Effectiveness Review No. 13. (Prepared by Minnesota Evidence-based Practice Center under Contract No. 290-202-0009). Rockville, MD: Agency for Healthcare Research and Quality; 2008. Available from: http://www.ncbi.nlm.nih.gov/books/NBK43147/pdf/TOC.pdf. Accessed January 20, 2011.

3. Davison BJ, Gleave ME, Goldenberg SL, Degner LF, Hoffart D, Berkowitz J. Assessing information and decision preferences of men with prostate cancer and their partners. Cancer Nurs. 2002;25(1):42-49.

4. Ramsey SD, Zeliadt SB, Hall IJ, Ekwueme DU, Penson DF. On the importance of race, socioeconomic status and comorbidity when evaluating quality of life in men with prostate cancer. $J$ Urol. 2007; 177(6):1992-1999.

5. Williams S, Hanson L, Boyd C, et al. Communication, decision making, and cancer: what African Americans want physicians to know. J Palliat Med. 2008;11(9):1221-1226.

6. Ramsey S, Zeliadt S, Fedorenko C, et al. Patient preferences and urologist recommendations among local-stage prostate cancer patients who present for initial consultation and second opinions. World J Urol. 2011; 29(1):3-9.

7. Brundage M, Feldman-Stewart D, Tishelman C. How do interventions designed to improve provider-patient communication work? Illustrative applications of a framework for communication. Acta Oncol. 2010;49(2):136-143.

8. Stewart MA. Effective physician-patient communication and health outcomes: a review. Can Med Assoc J. 1995;152(9):1423-1433.

9. Cohen H, Britten N. Who decides about prostate cancer treatment? A qualitative study. Fam Pract. 2003;20(6):724-729.

10. Cox J, Amling C. Current decision-making in prostate cancer therapy. Curr Opin Urol. 2008;18:275-278.

11. Emanuel EJ, Emanuel LL. Four models of the physician-patient relationship. JAMA. 1992;267(16):2221-2226.
12. Davison B, Degner L, Morgan T. Information and decision-making preferences of men with prostate cancer. Oncol Nurs Forum. 1995; 22(9): 1401-1408.

13. Davison BJ, Parker PA, Goldenberg SL. Patients' preferences for communicating a prostate cancer diagnosis and participating in medical decision-making. BJU Int. 2004;93(1):47-51.

14. Davison BJ, Degner L. Empowerment of men newly diagnosed with prostate cancer. Cancer Nurs. 1997;20(3):187-196.

15. Wong F, Stewart D, Dancey J, et al. Men with prostate cancer: influence of psychological factors on informational needs and decision making. J Psychosom Res. 2000;49:13-19.

16. Davison BJ, Goldenberg SL, Wiens KP, Gleave ME. Comparing a generic and individualized information decision support intervention for men newly diagnosed with localized prostate cancer. Cancer Nurs. 2007;30:7-15.

17. Stalmeier PF, van Tol-Geerdink JJ, van Lin EN, et al. Doctors' and patients' preferences for participation and treatment in curative prostate cancer radiotherapy. J Clin Oncol. 2007;25(21):3096-3100.

18. Van Tol-Geerdink J, Stalmeier P, van Lin E, et al. Do patients with localized prostate cancer treatment really want more aggressive treatment? J Clin Oncol. 2006;24(28):4581-4586.

19. Diefenbach MA, Mohamed NE. Regret of treatment decision and its association with disease-specific quality of life following prostate cancer treatment. Cancer Invest. 2007;25(6):449-457.

20. Boehmer U, Clark J. Communication about prostate cancer between men and their wives. J Fam Pract. 2001;50(3):226-231.

21. Burkhalter J, Bromberg S. Family-oncologist communication in cancer patient care. Cancer Invest. 2003;21(6):915-923.

22. Jones RA, Taylor AG, Bourguignon C, et al. Family interactions among African American prostate cancer survivors. Fam Community Health. 2008;31(3):213-220.
International Journal of General Medicine

\section{Publish your work in this journal}

The International Journal of General Medicine is an international, peer-reviewed open-access journal that focuses on general and internal medicine, pathogenesis, epidemiology, diagnosis, monitoring and treatment protocols. The journal is characterized by the rapid reporting of reviews, original research and clinical studies across all disease areas.

\section{Dovepress}

A key focus is the elucidation of disease processes and management protocols resulting in improved outcomes for the patient.The manuscript management system is completely online and includes a very quick and fair peer-review system. Visit http://www.dovepress.com/ testimonials.php to read real quotes from published authors. 